\title{
Use of slaughter condemnation data to detect cattle health events in near real-time
}

\author{
Judy Akkina, Leah Estberg
}

USDA, United States

Objective

Use United States cattle slaughter condemnation data as an animal health indicator for early detection of health events and to characterize trends in condemnation reasons.

\section{Introduction}

Data collected at livestock slaughter can be a useful source of non-specific health indicators including clinical signs, symptoms and proxy measures [1]. When monitored in near real-time, this data can enable the detection of both livestock and human health threats [1]. In the United States (US), the Federal Meat Inspection Act requires ante-mortem inspection of animals and post-mortem inspection of carcasses by veterinarians to ensure the meat product will be fit for human consumption [2]. Inspections are carried out by the United States Department of Agriculture (USDA) Food Safety Inspection Service (FSIS) and results are recorded in the Public Health Information System (PHIS), including the reason for condemnation if the animal or carcass is deemed unfit. Since April 2016 the USDA, Animal and Plant Health Inspection Service (APHIS), Veterinary Services (VS) has used data from the PHIS to monitor changes in the weekly count/rate of cattle condemnations for three cattle types, beef cows, dairy cows, and fed cattle (steers and heifers), and for selected condemnation reasons (central nervous system (CNS), dead, emaciation, mastitis, moribund, pneumonia, pyrexia, and septicemia). These eight reasons were chosen from 45 potential reasons recorded at condemnation as likely to identify clinical signs associated with foreign animal diseases of interest and to monitor trends in important animal health issues such as pneumonia and mastitis.

\section{Methods}

PHIS slaughter and condemnation data is downloaded weekly and stored and analyzed in an Access database. Tableau visualization software is used for mapping and time series signaling charts (example basin chart Figure 1). Only establishments slaughtering more than 600 cattle/week are included in analysis because smaller establishments may not operate weekly and many have very low slaughter volumes. With the small establishments excluded, our monitoring covers $93 \%, 90 \%$, and $85 \%$ of slaughtered beef, dairy, and fed cattle, respectively. Weekly analysis is conducted by cattle type for each included establishment and for establishments grouped into geographic based catchment basins. The basins were created to identify signals occurring over a region, even if at the establishment level the increase is not large enough to produce a signal. Another purpose for basins is to allow sharing of results at a summary level that does not identify an individual establishment. Weekly normalized condemnation counts are created by dividing the actual condemn counts by the total number destined for slaughter that week, and then multiplying by the average number destined for slaughter per week for the past year. The Early Aberration Reporting System (EARS C3) time series alerting algorithm is used to identify unusual increases (signals) in these weekly normalized condemnation counts. An analyst characterizes establishment and basin signals as unremarkable, notable or needing follow up with FSIS. An unremarkable signal is defined as at least one previous signal or normalized count of similar magnitude in the past 12 months and no signal for that establishment the previous week. A notable signal is defined as no previous signal or normalized count of similar magnitude in the past 12 months or two or more signals occurring over sequential weeks. Basin signals are researched to determine which establishments are responsible for the signal. An analyst determines which highly notable basin and establishment signals need follow up with FSIS. Results are summarized in a weekly report for VS cattle commodity staff about any noteworthy increases in condemnations which could indicate the emergence of disease and may warrant further investigation. In addition a 2017 annual report was completed to describe, visualize, summarize and compare condemnations from 2015-17 by cattle type and condemnation reason.

\section{Results}

In 2017 the percentage of beef, dairy and fed cattle condemned for the eight monitored reasons out of all cattle presented for slaughter at the monitored establishments was $0.375 \%, 1.651 \%$, and $0.022 \%$, respectively. For beef cows the three most frequent reasons were pneumonia, dead and septicemia, accounting for $84.3 \%$ of monitored condemnations. Cows that either arrive at the establishment dead or die at the establishment prior to slaughter are included in the dead condemnation count. For dairy cows the three most frequent reasons were dead, septicemia and pneumonia, accounting for $93.6 \%$ of condemnations. For fed cattle the three 
most frequent reasons were pneumonia, dead, and septicemia, accounting for $98.2 \%$ of condemnations. Beef cow condemnations exhibited consistent seasonal trends for dead, emaciation, mastitis and pneumonia. Dairy cow condemnations exhibited consistent seasonal trends for dead, emaciation and pyrexia. Fed cattle condemnations were less influenced by seasonal trends with only dead and septicemia showing some consistent seasonality. During 2017 three notable establishment signals required follow up with FSIS: CNS in dairy cows, septicemia in fed cattle, and dead in beef cows. For 2018 (through August) 5 notable establishment signals required follow up: septicemia in both beef and dairy cows, emaciation and CNS in beef cows, and dead in dairy cows. Signals were attributed to various reasons including: changes in establishment protocol (CNS), relief inspectors classifying condemns differently and animals not responding to treatment on the farm (septicemia), a holiday effect with sick animals held longer in holding pens before slaughter (dead), a decrease in the quality of cattle purchased by the establishment (emaciation), and extremely hot weather (dead).

\section{Conclusions}

A higher percentage of dairy cattle were condemned overall and were more likely to be condemned for being dead on arrival or just prior to slaughter compared to beef or fed cattle. Culled dairy cows tend to be older than beef or fed cattle and are maintained in an intense production system, therefore they are more likely to develop age related problems or chronic diseases [3]. In addition to timely identification of potential animal health issues, cattle producers could use this information to help focus on management practices which may decrease condemnations and result in improved animal health and revenue for producers [2]. Some limitations to this work are that trends described only apply to the cattle population slaughtered at FSIS inspected facilities and included in our monitoring. Condemnations are determined by FSIS inspectors at each individual slaughter establishment resulting in the potential for inspector bias to affect the data.

\section{References}

1.Dupuy C, Morignat E, Dorea F, Cucrot C, Calavas D, et al. 2015. Pilot simulation study using meat inspection data for syndromic surveillance: use of whole carcass condemnation of adult cattle to assess the performance of several algorithms for outbreak detection. Epidemiol Infect. 143(12), 2559-69. PubMed https://doi.org/10.1017/S0950268814003495

2. White T, Moore D. (2009) Reasons for whole carcass condemnations of cattle in the United States and implications for producer education and veterinary intervention. JAVMA Oct;235(8).

3. Haredasht S, Vidal G, Edmondson A, Moore D, Silva-del-Rio N, Martinez-Lopez B. (2018) Characterization of the temporal trends in the rate of cattle carcass condemnation in the US and dynamic modeling of the condemnation reasons in California with a seasonal component. Front Vet Sci Jun;5(87).

Figure 1

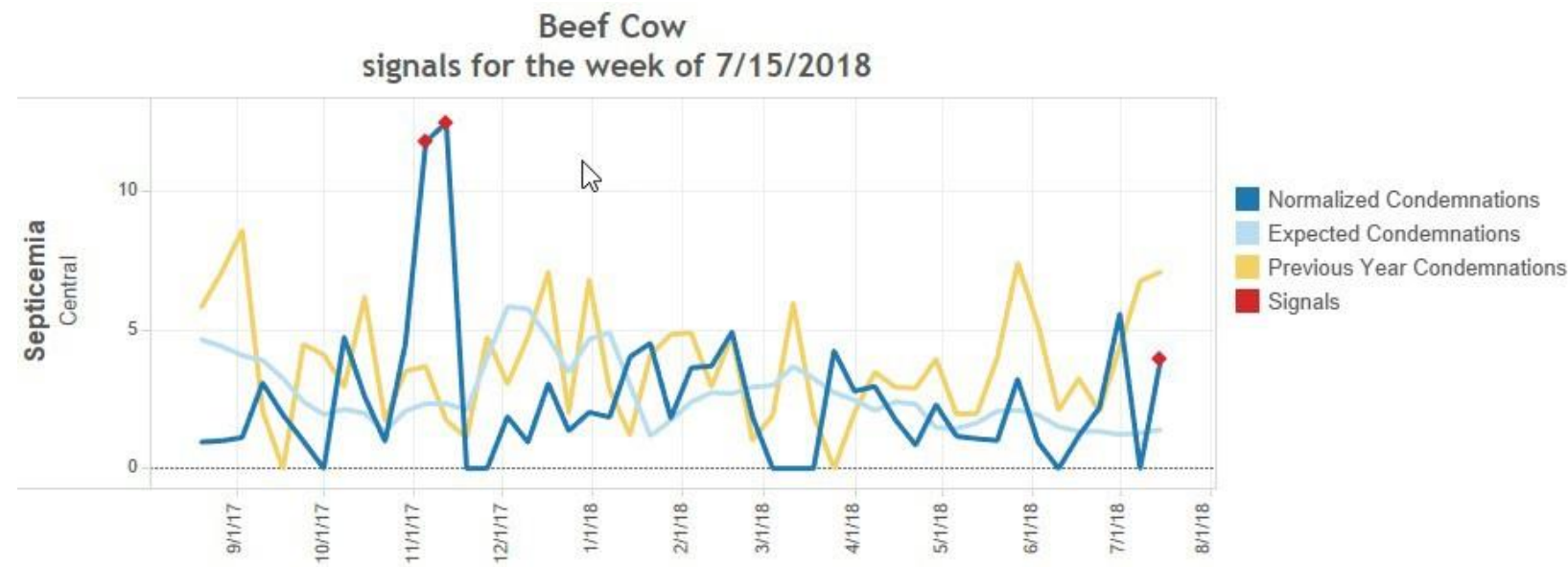

ISDS Annual Conference Proceedings 2019. This is an Open Access article distributed under the terms of the Creative Commons AttributionNoncommercial 4.0 Unported License (http://creativecommons.org/licenses/by-nc/3.0/), permitting all non-commercial use, distribution, and reproduction in any medium, provided the original work is properly cited. 